\title{
Privacy-Utility Tradeoff in a Guessing Framework Inspired by Index Coding
}

\author{
Yucheng Liu ${ }^{\dagger}$, Ni Ding*, Parastoo Sadeghi ${ }^{\dagger}$, and Thierry Rakotoarivelo* \\ ${ }^{\dagger}$ Research School of Electrical, Energy and Materials Engineering, Australian National University, Australia \\ ${ }^{*}$ Data61, Commonwealth Scientific and Industrial Research Organisation, Australia \\ Emails: ${ }^{\dagger}\{$ yucheng.liu, parastoo.sadeghi $\} @$ anu.edu.au, ${ }^{*}\{$ ni.ding, thierry.rakotoarivelo $\} @$ data61.csiro.au
}

\begin{abstract}
This paper studies the tradeoff in privacy and utility in a single-trial multi-terminal guessing (estimation) framework using a system model that is inspired by index coding. There are $n$ independent discrete sources at a data curator. There are $m$ legitimate users and one adversary, each with some side information about the sources. The data curator broadcasts a distorted function of sources to legitimate users, which is also overheard by the adversary. In terms of utility, each legitimate user wishes to perfectly reconstruct some of the unknown sources and attain a certain gain in the estimation correctness for the remaining unknown sources. In terms of privacy, the data curator wishes to minimize the maximal leakage: the worst-case guessing gain of the adversary in estimating any target function of its unknown sources after receiving the broadcast data. Given the system settings, we derive fundamental performance lower bounds on the maximal leakage to the adversary, which are inspired by the notion of confusion graph and performance bounds for the index coding problem. We also detail a greedy privacy enhancing mechanism, which is inspired by the agglomerative clustering algorithms in the information bottleneck and privacy funnel problems.
\end{abstract}

\section{INTRODUCTION}

In this paper, we consider an information-theoretic multiterminal guessing framework with side information. Our model is inspired by that of the index coding problem [1], [2], but with a significant twist to place emphasis on privacy. Index coding is a communication problem where a sender aims to efficiently broadcast to multiple users through a noiseless channel. See [3] and references therein. In particular, the security and privacy aspects of index coding have been investigated in [4]-[10]. Instead of maximizing the communication rate, in our framework the sender's goal is to balance the privacy and utility performance in the broadcast, both measured based on the success rate of correctly estimating a certain parameter of interest about the sources through a single guess. We consider multiple independent sources available at a data curator and assume that there are multiple legitimate users, as well as one adversary in the system. Each party (a user or the adversary) knows some sources a priori as side information. The data curator broadcasts (discloses) a distorted function of the sources to the users, which is also overheard by the adversary. Such framework has applications in various real-world scenarios, such as field data broadcasting from a paddock aggregator in the presence of a malicious agent in precision agriculture.

For the adversary, we adopt the maximal leakage introduced in [11] as the privacy metric. It measures the worstcase information leakage in terms of the gain of the adversary in maximum a posteriori estimation of any target function of the unknown sources after and before observing the disclosed data. For legitimate users, we define our utility metric such that it also reflects the improvement in users' guessing ability. Quite often in practice different sources are of different levels of priority to a user. We capture this by dividing the unknown sources of each user into two subsets. Some essential source are required to be perfectly reconstructed by the user. That is, the correct guessing probability of such sources after observing the disclosed data is non-negotiable and must be 1. The remaining sources are less critical, for which the user requires the guessing gain about each such source to be larger than a certain negotiable threshold. The privacyutility tradeoff is cast as a constrained optimization problem, where the objective is to minimize the privacy leakage to the adversary, conditioned that the requirements on the utility of the unknown sources for the legitimate users are satisfied.

The contributions of this paper are twofold. First, we derive two lower bounds on the privacy leakage given the source distribution and utility constraints (cf. Theorems 1 . 2) from different persepctives. These lower bounds serve as converse results (fundamental performance limits) for the privacy leakage. Second, we propose a greedy algorithm as the privacy mechanism (cf. Algorithm 11) inspired by the agglomerative clustering method used in the information bottleneck and privacy funnel problems [12]-[14]. We leverage the connection between our data disclosure problem and the index coding problem when investigating both the converse results and the greedy algorithm.

Notation: For non-negative integers $a$ and $b,[a]$ denotes the set $\{1,2, \cdots, a\}$ and $[a: b]$ denotes the set $\{a, a+1, \cdots, b\}$. For a set $S,|S|$ denotes its cardinality. For any discrete random variable $Z$ with probability distribution $P_{Z}$, we denote its alphabet by $\mathcal{Z}$ with realizations $z \in \mathcal{Z}$. We denote an estimator of $Z$ by $\hat{Z}$, whose alphabet is also $\mathcal{Z}$.

\section{System Model And PRoblem Formulation}

Assume that a data curator observes $n$ independent discrete random sources $X_{1}, X_{2}, \ldots, X_{n}$. We assume a general distribution $P_{X_{i}}$ for each source. Without loss of generality, we assume every source has full support. For brevity, when we say source $i$, we mean source $X_{i}$. For any $S \subseteq[n]$, set $S^{c}=[n] \backslash S, X_{S}=\left(X_{i}: i \in S\right), x_{S}=\left(x_{i}: i \in S\right)$, and $\mathcal{X}_{S}=\prod_{i \in S} \mathcal{X}_{i}$. The data curator broadcasts a distorted version of $X_{[n]}$, denoted by $Y$, generated according to the 
privacy mechanism $P_{Y \mid X_{[n]}}$, to a number of legitimate users, which is also overheard by an adversary.

We consider a guessing framework where both the legitimate users and the adversary attempt to estimate a certain parameter $V$ of interest about the sources in a single trial.

Both the privacy and utility are measured in terms of a guessing gain $r$ as defined below. Consider any party (a user or the adversary) that wishes to guess $V$ with the side information $Z$. Note that $V \Perp Z$ due to source independence. The party aims to maximize the correct guessing probability of $V$ upon observing $Y$ (i.e., the party employs the maximum a posteriori estimator). For each $(y, z) \in \mathcal{Y} \times \mathcal{Z}$, we define the ratio between such maximized guessing probability after and before observing a $y \in \mathcal{Y}$ given $z \in \mathcal{Z}$ as

$$
\begin{aligned}
r(V \rightarrow y \mid z) \doteq & \frac{\max _{\hat{V} \mid Y=y, Z=z} \mathbb{E}\left[P_{\hat{V} \mid Y=y, Z=z}(V \mid y, z)\right]}{\max _{P_{\hat{V} \mid Z=z}} \mathbb{E}\left[P_{\hat{V} \mid Z=z}(V \mid z)\right]} \\
& =\frac{\max _{v} P_{V \mid Y, Z}(v \mid y, z)}{\max _{v} P_{V}(v)} .
\end{aligned}
$$

Privacy Metric: We assume the adversary has side information $X_{P}$ for some $P \subseteq[n]$, and is interested in a (possibly randomized) discrete function $U$ of the sources $X_{Q}$ it does not know, where $Q \doteq P^{c}$. Quite often in practice, this function is chosen by the adversary and is unknown to the data curator. Therefore, we consider a worst-case privacy leakage measure, a conditional version of maximal leakage (MaxL) from [11], as our privacy metric.

Definition 1 (Maximal Leakage, [11]): Given a finite discrete joint distribution $P_{X_{[n]}, Y}$, the maximal leakage from $X_{Q}$ to $\mathrm{Y}$ given $X_{P}$ is defined as

$$
\mathcal{L}_{\max }\left(X_{Q} \rightarrow Y \mid X_{P}\right) \doteq \sup _{U: U-X_{Q}-\left(Y, X_{P}\right)} \mathcal{L}\left(U \rightarrow Y \mid X_{P}\right),
$$

where

$$
\mathcal{L}\left(U \rightarrow Y \mid X_{P}\right) \doteq \log \mathbb{E}_{P_{Y, X_{P}}}\left[r\left(U \rightarrow Y \mid X_{P}\right)\right] .
$$

Remark 1: Note that the MaxL in Definition 1 assumes a different Markov chain model from [11, Section III-E]: for our problem, the Markov chain model studied in [11] always reduces to $U-X_{[n]}-Y$ regardless of $Q$.

For the rest of the paper, we refer to $\mathcal{L}_{\max }\left(X_{Q} \rightarrow Y \mid X_{P}\right)$ as $\mathcal{L}_{\max }$ when there is no ambiguity. A computable expression of the MaxL in Definition 1 is presented as

$$
\mathcal{L}_{\text {max }}=\log \sum_{y, x_{P}} \max _{x_{Q}} P_{Y, X_{P} \mid X_{Q}}\left(y, x_{P} \mid x_{Q}\right),
$$

which can be obtained following a similar approach to [11]. We omit the derivation details due to limited space.

Utility Constraints: There are $m$ legitimate users. User $i \in[m]$ knows some sources $X_{A_{i}}$ a priori as side information for some $A_{i} \subseteq[n]$, and is interested in all the remaining sources $X_{A_{i}^{c}}$, which are divided into two groups of different levels of priority:

- Source $X_{W_{i}}$ for some $W_{i} \subseteq A_{i}^{c}$ are indispensable to the user, and thus must be correctly guessed by user $i$ with probability of 1 (i.e., perfect decoding).
- The rest of the sources, $X_{G_{i}}$, where $G_{i} \doteq A_{i}^{c} \backslash W_{i}$, are less essential yet still useful/interesting. Thus, user $i$ requires the guessing ability gain upon observing $Y$ to be larger than a certain threshold $d_{i}$.

These result in the following two kinds of utility constraints. For any $i \in[m]$, we have

$$
\begin{array}{ll}
H\left(X_{W_{i}} \mid Y, X_{A_{i}}\right)=0, & \forall i \in[m], \\
D\left(X_{G_{i}} \rightarrow Y \mid X_{A_{i}}\right) \geq d_{i}, & \forall i \in[m],
\end{array}
$$

where $D\left(X_{G_{i}} \rightarrow Y \mid X_{A_{i}}\right)$ is defined as

$$
D\left(X_{G_{i}} \rightarrow Y \mid X_{A_{i}}\right) \doteq \mathbb{E}_{P_{Y, X_{A_{i}}}}\left[\log r\left(X_{G_{i}} \rightarrow Y \mid X_{A_{i}}\right)\right] .
$$

Note that for constraint (7), each legitimate user $i$ is interested in obtaining the source $X_{G_{i}}$ rather than a function/feature of $X_{G_{i}}$. We simplify the notation $D\left(X_{G_{i}} \rightarrow\right.$ $\left.Y \mid X_{A_{i}}\right)$ to $D_{i}$ when there is no ambiguity.

Remark 2: Note the subtle difference between $D_{i}$ and $\mathcal{L}\left(X_{G_{i}} \rightarrow Y \mid X_{A_{i}}\right)=\log \mathbb{E}_{P_{Y, X_{A_{i}}}}\left[r\left(X_{G_{i}} \rightarrow Y \mid X_{A_{i}}\right)\right]$ as in (4). The latter is lower bounded by the former due to Jensen's inequality. From the data curator's viewpoint, requesting $D_{i}$ to be above a certain threshold is more stringent than requesting $\mathcal{L}\left(X_{G_{i}} \rightarrow Y \mid X_{A_{i}}\right)$ to be above that threshold. We use $D_{i}$ rather than $\mathcal{L}\left(X_{G_{i}} \rightarrow Y \mid X_{A_{i}}\right)$ as our utility measure as it leads to a simple closed-form result characterizing $\mathcal{L}_{\text {max }}$ in terms of $D_{i}$ (cf. Lemma 2).

Remark 3: The two types of utility constraints in (6) and (7) can be unified to be represented in terms of the same function $D$ : The perfect decoding constraint (6) is equivalent to requiring that

$$
\begin{aligned}
D\left(X_{W_{i}} \rightarrow Y \mid X_{A_{i}}\right) & =\mathbb{E}_{P_{Y, X_{A_{i}}}}\left[\log \frac{1}{\max _{x_{W_{i}}} P_{X_{W_{i}}}\left(x_{W_{i}}\right)}\right] \\
& =H_{\infty}\left(X_{W_{i}}\right)
\end{aligned}
$$

where $H_{\infty}\left(X_{W_{i}}\right)$ denotes the min-entropy (i.e., Rényi entropy of order $\infty$ [15]) of $X_{W_{i}}$. One can show that for any $i \in[m], D_{i} \leq H_{\infty}\left(X_{G_{i}}\right)$. Consequently, we always require that $0 \leq d_{i} \leq H_{\infty}\left(X_{G_{i}}\right)$ for any $i \in[m]$.

Privacy-Utility Tradeoff: We denote such system by the 5-tuple $\left(P_{X_{[n]}}, \mathbf{A}, \mathbf{W}, \mathbf{d}, P\right)$, where $\mathbf{A} \doteq\left(A_{i}, i \in[m]\right)$, $\mathbf{W} \doteq\left(W_{i}, i \in[m]\right)$, and $\mathbf{d} \doteq\left(d_{i}, i \in[m]\right)$. Note that $G_{i}$ is determined by $W_{i}$ and $A_{i}$, and $Q$ is determined by $P$.

To design the privacy mechanism $P_{Y \mid X_{[n]}}$, we need to consider the fundamental tradeoff between the privacy and utility. Any data distortion that reduces the information leakage to the adversary can decrease the utility obtained by the users. Such tradeoff is formulated by the following constrained optimization problem.

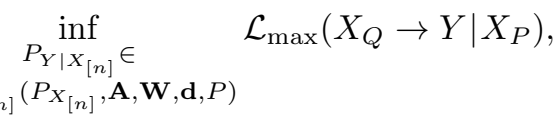

where $\mathcal{P}_{Y \mid X_{[n]}}\left(P_{X_{[n]}}, \mathbf{A}, \mathbf{W}, \mathbf{d}, P\right)$ denotes the collection of randomized mappings $P_{Y \mid X_{[n]}}$ that satisfy (6) and (7) for the problem $\left(P_{X_{[n]}}, \mathbf{A}, \mathbf{W}, \mathbf{d}, P\right)$.

Due to the non-convexity of (10), instead of providing an explicit solution, we derive lower bounds on $\mathcal{L}_{\max }$ by 
taking inspiration from index coding. These bounds serve as fundamental performance limits that cannot be surpassed by any mechanism because they are enforced by the system $\left(P_{X_{[n]}}, \mathbf{A}, \mathbf{W}, \mathbf{d}, P\right)$. In Section IV we design an achievable mechanism based on the idea of agglomerative clustering.

\section{LOWER Bounds ON THE PRIVACY LEAKAGE}

We derive two information-theoretic lower bounds on the privacy leakage. One is based on the utility constraint (6) only, while the other is obtained based on both (6) and (7).

\section{A. Lower Bound Based on the Confusion Graph}

The utility constraint (6) indicates that for user $i$, any different realizations $x_{W_{i}} \neq x_{W_{i}}^{\prime} \in \mathcal{X}_{W_{i}}$ must be distinguishable based on the released $y \in \mathcal{Y}$, as well as the user's side information $x_{A_{i}} \in \mathcal{X}_{A_{i}}$. To describe such distinguishability, we recall the notion of confusion graph for index coding [16].

Definition 2 (Confusion graph [16]): Any two realizations of the $n$ sources $x_{[n]}^{1}, x_{[n]}^{2} \in \mathcal{X}_{[n]}$ are confusable if there exists some user $i \in[m]$ such that $x_{W_{i}}^{1} \neq x_{W_{i}}^{2}$ and $x_{A_{i}}^{1}=x_{A_{i}}^{2}$. A confusion graph $\Gamma$ is an undirected uncapacitated graph with $\left|\mathcal{X}_{[n]}\right|$ vertices such that every vertex corresponds to a unique realization $x_{[n]} \in \mathcal{X}_{[n]}$ and an edge connects two vertices if and only if their corresponding realizations are confusable.

Therefore, to ensure (6), a group of realizations of $X_{[n]}$ can be mapped to the same $y$ with nonzero probability only if they are pairwisely not confusable. More rigorously, for any $S \subseteq[n]$, define

$$
\begin{array}{ll}
\mathcal{Y}\left(x_{S}\right) \doteq\left\{y \in \mathcal{Y}: P_{Y \mid X_{S}}\left(y \mid x_{S}\right)>0\right\}, & \forall x_{S} \in \mathcal{X} \\
\mathcal{X}_{S}(y) \doteq\left\{x_{S} \in \mathcal{X}_{S}: P_{Y \mid X_{S}}\left(y \mid x_{S}\right)>0\right\}, & \forall y \in \mathcal{Y} .
\end{array}
$$

Then, we have the following lemma. We omit the proof as it can be simply done by contradiction.

Lemma 1: Given a $P_{Y \mid X_{[n]}}$ satisfying (6), for any two confusable $x_{[n]}^{1}, x_{[n]}^{2} \in \mathcal{X}_{[n]}$, we have $\mathcal{Y}\left(x_{[n]}^{1}\right) \cap \mathcal{Y}\left(x_{[n]}^{2}\right)=\emptyset$.

Given a set $S \subseteq[n]$ and a specific realization $x_{S} \in \mathcal{X}_{S}$, we define $\Gamma\left(x_{S}\right)$ as the subgraph of $\Gamma$ induced by all the vertices $x_{[n]}$ such that $x_{[n]}=\left(x_{S}, x_{S^{c}}\right)$ for some $x_{S^{c}} \in$ $\mathcal{X}_{S^{c}}$. Notice that for any $x_{S}^{1} \neq x_{S}^{2} \in \mathcal{X}_{S}$ and $x_{S^{c}}^{1} \neq x_{S^{c}}^{2} \in$ $\mathcal{X}_{S^{c}},\left(x_{S}^{1}, x_{S^{c}}^{1}\right)$ and $\left(x_{S}^{1}, x_{S^{c}}^{2}\right)$ are confusable if and only if $\left(x_{S}^{2}, x_{S^{c}}^{1}\right)$ and $\left(x_{S}^{2}, x_{S^{c}}^{2}\right)$ are confusable. Hence, given $S \subseteq$ $[n]$, the subgraphs $\Gamma\left(x_{S}\right), x_{S} \in \mathcal{X}_{S}$ are isomorphic to each other, and thus we simply denote any such subgraph by $\Gamma(S)$.

We present our main result of this subsection as follows.

Theorem 1: For the problem 10] with confusion graph $\Gamma$ :

$$
\mathcal{L}_{\max } \geq \log \omega(\Gamma(P)),
$$

where $\omega(\cdot)$ is the clique number (size of the largest clique) of a graph.

Proof: Consider any $x_{P} \in \mathcal{X}_{P}$. There exists some realizations $x_{Q}^{1}, x_{Q}^{2}, \ldots, x_{Q}^{\omega\left(\Gamma\left(x_{P}\right)\right)} \in \mathcal{X}_{Q}$ whose corresponding vertices in the subgraph $\Gamma\left(x_{P}\right)$ form a clique, which indicates that the realizations $\left(x_{P}, x_{Q}^{1}\right),\left(x_{P}, x_{Q}^{2}\right), \ldots,\left(x_{P}, x_{Q}^{\omega\left(\Gamma\left(x_{P}\right)\right)}\right)$ also form a clique in $\Gamma$. That is, the realizations $\left(x_{P}, x_{Q}^{1}\right), \ldots,\left(x_{P}, x_{Q}^{\omega\left(\Gamma\left(x_{P}\right)\right)}\right)$ are pairwisely confusable. Then by Lemma 1, for any $k \neq k^{\prime} \in\left[\omega\left(\Gamma\left(x_{P}\right)\right)\right]$, we have

$$
\mathcal{Y}\left(\left(x_{P}, x_{Q}^{k}\right)\right) \cap \mathcal{Y}\left(\left(x_{P}, x_{Q}^{k^{\prime}}\right)\right)=\emptyset .
$$

Therefore, we have

$$
\begin{aligned}
& \sum_{y} \max _{x_{Q}} P_{Y \mid X_{[n]}}\left(y \mid x_{P}, x_{Q}\right) \\
& \geq \sum_{k \in\left[\omega\left(\Gamma\left(x_{P}\right)\right)\right]} \sum_{y \in \mathcal{Y}\left(x_{P}, x_{Q}^{k}\right)} \max _{x_{Q}} P_{\left.Y \mid X_{[n]}\right]}\left(y \mid x_{P}, x_{Q}\right) \\
& \geq \sum_{k \in\left[\omega\left(\Gamma\left(x_{P}\right)\right)\right]} \sum_{y \in \mathcal{Y}\left(x_{P}, x_{Q}^{k}\right)} P_{Y \mid X_{[n]}}\left(y \mid x_{P}, x_{Q}^{k}\right) \\
& =\sum_{k \in\left[\omega\left(\Gamma\left(x_{P}\right)\right)\right]} 1=\omega\left(\Gamma\left(x_{P}\right)\right)=\omega(\Gamma(P)),
\end{aligned}
$$

where the first inequality follows from (12). Hence, we have

$$
\begin{aligned}
\mathcal{L}_{\max } & =\log \sum_{x_{P}} P_{X_{P}}\left(x_{P}\right) \sum_{y \in \mathcal{Y}} \max _{x_{Q}} P_{Y \mid X_{[n]}}\left(y \mid x_{P}, x_{Q}\right) \\
& \geq \log \sum_{x_{P}} P_{X_{P}}\left(x_{P}\right) \cdot \omega(\Gamma(P))=\log \omega(\Gamma(P)),
\end{aligned}
$$

where the first equality is due to source independence, and the inequality follows from (13).

\section{B. Lower Bound Based on Guessing Gain and Polymatroidal Functions}

We introduce a key lemma that serves as the baseline in the lower bound to be developed in this subsection.

Lemma 2: For the problem (10), we have

$$
\mathcal{L}_{\max } \geq \max \left\{I\left(X_{Q} ; Y \mid X_{P}\right), \max _{i \in[m]: G_{i} \subseteq Q} \Delta_{i}\right\}
$$

where $\Delta_{i} \doteq D_{i}+H\left(X_{W_{i} \cap Q}\right)+I\left(X_{A_{i} \cap Q} ; Y \mid X_{P}\right)$.

The proof is presented in Appendix A

For a given system $\left(P_{X_{[n]}}, \mathbf{A}, \mathbf{W}, \mathbf{d}, P\right), H\left(X_{W_{i} \cap Q}\right)$ has a fixed value and $D_{i}$ is lower bounded by $d_{i}$ according to (7). Hence, the only terms in (14) that still depend on $P_{Y \mid X_{[n]}}$ are the mutual information $I\left(X_{P} ; Y \mid X_{Q}\right)$ and $I\left(X_{A_{i} \cap Q} ; Y \mid X_{P}\right)$. We further bound these mutual information terms below.

We draw inspiration from the polymatroidal bound [17], [18] for index coding. The bound is based on the polymatroidal axioms, which capture Shannon-type inequalities on the entropy function and play a central role in computing converse results in network information theory [19].

Lemma 3: Consider the system $\left(P_{X_{[n]}}, \mathbf{A}, \mathbf{W}, \mathbf{d}, P\right)$. For any disjoint $V, Z \subseteq[n]$, we have

$$
I\left(X_{V} ; Y \mid X_{Z}\right)=g\left(Z^{c}\right)-g\left(Z^{c} \cap V^{c}\right),
$$

for some polymatroidal rank function $g(S), S \subseteq[n]$ such that for any $i \in[n], W \subseteq W_{i}, G \subseteq W^{c} \cap A_{i}^{c}$,

$$
H\left(X_{W}\right)=g(G \cup W)-g(G),
$$

and

$$
\begin{aligned}
& g(\emptyset)=0, \\
& g\left(S^{\prime}\right) \geq g(S), \quad \text { if } S \subseteq S^{\prime}, \\
& g\left(S^{\prime}\right)+g(S) \geq g\left(S^{\prime} \cup S\right)+g\left(S^{\prime} \cap S\right) .
\end{aligned}
$$

Proof: Define $g(S) \doteq H\left(Y \mid X_{S^{c}}\right)-H\left(Y \mid X_{[n]}\right), \forall S \subseteq$ $[n]$. We have $I\left(X_{V} ; Y \mid X_{Z}\right)=g\left(Z^{c}\right)-g\left(Z^{c} \cap V^{c}\right)$. It remains to show that this $g(S)$ satisfies (16)-(19). 
For (16), consider any $i \in[n], W \subseteq W_{i}, G \subseteq W^{c} \cap A_{i}^{c}$. Set $A=[n] \backslash W \backslash G$, and one can verify that $A_{i} \subseteq A$. Hence,

$$
\begin{aligned}
H\left(X_{W}\right) & =H\left(X_{W} \mid X_{A}\right)-H\left(X_{W} \mid Y, X_{A}\right) \\
& =H\left(Y \mid X_{A}\right)-H\left(Y \mid X_{W}, X_{A}\right) \\
& =g(W, G)-g(G),
\end{aligned}
$$

where (20) is due to source independence, (6), and $A_{i} \subseteq A$.

For (17), we have $g(\emptyset)=H\left(Y \mid X_{[n]}\right)-H\left(Y \mid X_{[n]}\right)=0$.

For (18), for any $S \subseteq S^{\prime} \subseteq[n], S^{\prime c} \subseteq S^{c}$, and thus

$$
g\left(S^{\prime}\right)=H\left(Y \mid X_{S^{\prime c}}\right) \geq H\left(Y \mid X_{S^{c}}\right)=g(S) .
$$

For [19], consider any $S, S^{\prime} \subseteq[n]$. Set $S^{c} \cap S^{\prime c}=S_{0}$, $S^{c} \backslash S_{0}=S_{1}$, and $S^{\prime c} \backslash S_{0}=S_{2}$. We have

$$
\begin{aligned}
& g\left(S^{\prime}\right)+g(S) \\
& =H\left(Y, X_{S_{0} \cup S_{2}}\right)+H\left(Y, X_{S_{0} \cup S_{1}}\right)-H\left(X_{S_{0} \cup S_{2}}\right)-H\left(X_{S_{0} \cup S_{1}}\right) \\
& \geq H\left(Y, X_{S_{0}}\right)+H\left(Y, X_{S_{0} \cup S_{1} \cup S_{2}}\right)-H\left(X_{S_{0}}\right)-H\left(X_{S_{0} \cup S_{1} \cup S_{2}}\right) \\
& =g\left(S \cup S^{\prime}\right)+g\left(S \cap S^{\prime}\right),
\end{aligned}
$$

where the inequality follows from the submodularity of the entropy function, as well as source independence.

The above bound can be solved using either linear programming (LP) or Fourier-Motzkin elimination [20, Appendix D]. In the system $\left(P_{X_{[n]}}, \mathbf{A}, \mathbf{W}, \mathbf{d}, P\right)$, for any disjoint $V, Z \subseteq[n]$, let

$$
\Lambda(V, Z) \doteq \min _{g \text { satisfying [16-[19] }}\left\{g\left(Z^{c}\right)-g\left(Z^{c} \cap V^{c}\right)\right\} .
$$

Combining Lemmas 2 and 3 gives the following result.

Theorem 2: For the problem (10), we have

$$
\begin{aligned}
& \mathcal{L}_{\max } \geq \max \{\Lambda(Q, P), \\
& \left.\max _{i \in[m]: G_{i} \subseteq Q}\left(d_{i}+H\left(X_{W_{i} \cap Q}\right)+\Lambda\left(A_{i} \cap Q, P\right)\right)\right\} .
\end{aligned}
$$

Remark 4: In the index coding problem, we usually assume uniformly distributed independent sources and a deterministic mapping $P_{Y \mid X_{[n]}}$. In contrast, Theorems 1 and 2 generally hold for any discrete independent source distribution and make no assumption on the privacy mechanism.

In general, Theorems 1 and 2 can outperform each other.

\section{Privacy Mechanism Design}

We develop a greedy algorithm to provide a solution for the problem (10). The algorithm is based on the agglomerative clustering method, which has been used in the information bottleneck [12] and the privacy funnel problem [13].

Consider a given system $\left(P_{X_{[n]}}, \mathbf{A}, \mathbf{W}, \mathbf{d}, P\right)$. To design the privacy mechanism $P_{Y \mid X_{[n]}}$, we start from the one-to-one deterministic mapping with $\mathcal{Y}=\mathcal{X}_{[n]}$ and $P_{Y \mid X_{[n]}}\left(y \mid x_{[n]}\right)=$ 1 iff $\left.y=x_{[n}\right] 1$ and then iteratively merge some elements of $\mathcal{Y}$ to make the privacy leakage smaller (in other words, we "blur" the revealed information), while still ensuring the utility for the users at an acceptable level, i.e., satisfying [6]

\footnotetext{
${ }^{1}$ Note that such one-to-one mapping allows every user to perfectly reconstruct every source and thus satisfies $(6)$ and $(7)$ for sure. Nevertheless, it also leads to the largest privacy leakage as the adversary can also perfectly reconstruct $X_{Q}$ and subsequently any function $U$ it is interested in.
}

and (7). In particular, to ensure (6), we again utilize the notion of confusion graph [16] introduced in Section [II-A

Based on the merging idea discussed above, we propose an agglomerative clustering algorithm in Algorithm 1 Let $Y^{y_{1}, y_{2}}$ be the resulting random variable from merging any $y_{1}, y_{2} \in \mathcal{Y}$. Let $\Theta$ denote the collection of $\left\{y_{1}, y_{2}\right\}$ such that merging them does not violate (6) and (7) and strictly reduces the privacy leakage to the adversary:

$$
\begin{aligned}
\Theta \doteq & \left\{y_{1}, y_{2}\right\} \in \mathcal{Y} \times \mathcal{Y}: y_{1} \neq y_{2}, \text { any two } \\
& x_{[n]}, x_{[n]}^{\prime} \in \mathcal{X}_{[n]}\left(y_{1}\right) \cup \mathcal{X}_{[n]}\left(y_{2}\right) \text { are not confusable, } \\
& D\left(X_{G_{i}} \rightarrow Y^{y_{1}, y_{2}} \mid X_{A_{i}}\right) \geq d_{i}, \forall i \in[m], \\
& \left.\mathcal{L}_{\max }\left(X_{Q} \rightarrow Y^{y_{1}, y_{2}} \mid X_{P}\right)<\mathcal{L}_{\max }\left(X_{Q} \rightarrow Y \mid X_{P}\right)\right\} .
\end{aligned}
$$

The algorithm terminates if $\Theta$ becomes an empty set.

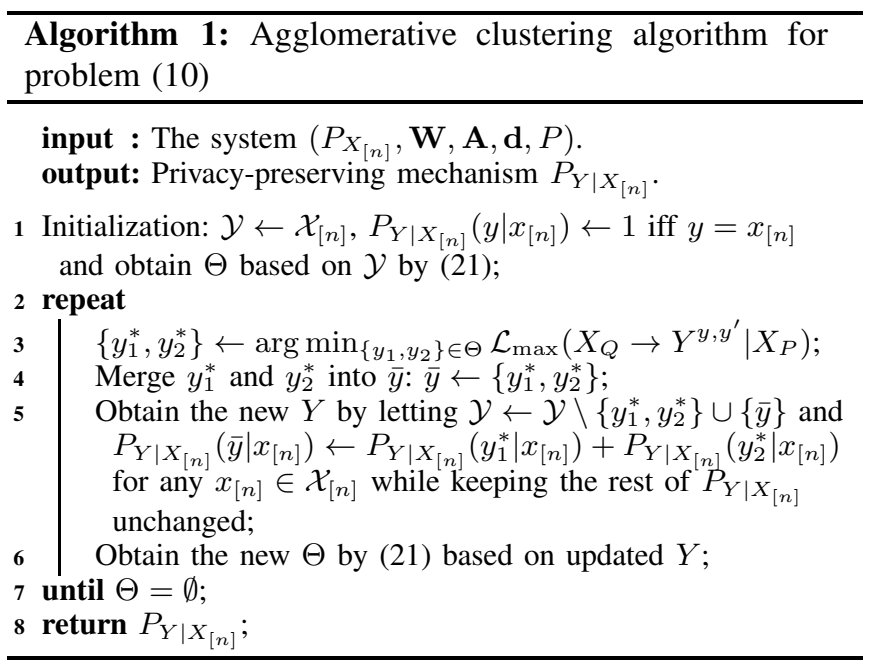

Remark 5: To compute Algorithm 1 more efficiently, notice that finding $\arg \min _{\left\{y_{1}, y_{2}\right\} \in \Theta} L_{\max }\left(X_{Q} \rightarrow Y^{y_{1}, y_{2}} \mid X_{P}\right)$ is equivalent to finding arg $\max _{\left\{y_{1}, y_{2}\right\} \in \Theta}\left(2^{\mathcal{L}_{\max }\left(X_{Q} \rightarrow Y \mid X_{P}\right)}-\right.$ $2^{\left.\mathcal{L}_{\max }\left(X_{Q} \rightarrow Y^{y_{1}, y_{2}} \mid X_{P}\right)\right)}$ in step 3, which can be computed as

$$
\begin{aligned}
& 2^{\mathcal{L}_{\max }\left(X_{Q} \rightarrow Y \mid X_{P}\right)}-2^{\mathcal{L}_{\max }\left(X_{Q} \rightarrow Y^{y_{1}, y_{2}} \mid X_{P}\right)} \\
&= \sum_{x_{P}} \max _{x_{Q}} P_{X_{P}}\left(x_{P}\right) \cdot P_{Y \mid X_{[n]}}\left(y_{1} \mid x_{P}, x_{Q}\right) \\
&+\sum_{x_{P}} \max _{x_{Q}} P_{X_{P}}\left(x_{P}\right) \cdot P_{Y \mid X_{[n]}}\left(y_{2} \mid x_{P}, x_{Q}\right) \\
&-\sum_{x_{P}} \max _{x_{Q}} P_{X_{P}}\left(x_{P}\right) \cdot P_{Y \mid X_{[n]}}\left(\bar{y} \mid x_{P}, x_{Q}\right) \\
&= \sum_{x_{P} \in \mathcal{X}_{P}\left(y_{1}\right)} P_{X_{P}}\left(x_{P}\right) \cdot 1+\sum_{x_{P} \in \mathcal{X}_{P}\left(y_{2}\right)} P_{X_{P}}\left(x_{P}\right) \cdot 1 \\
&-\sum_{x_{P} \in \mathcal{X}_{P}(\bar{y})} P_{X_{P}}\left(x_{P}\right) \cdot 1 \\
&= P_{X_{P}}\left(\mathcal{X}_{P}\left(y_{1}\right)\right)+P_{X_{P}}\left(\mathcal{X}_{P}\left(y_{2}\right)\right)-P_{X_{P}}\left(\mathcal{X}_{P}(\bar{y})\right) .
\end{aligned}
$$

\section{CONCluding REMARKS}

To evaluate the performance of our main results, we randomly generate the system $\left(P_{X_{[n]}}, \mathbf{A}, \mathbf{W}, \mathbf{d}, P\right)$ by 500 times according to the following conditions:

- $n=m=5, W_{i}=\{i\}$ for any user $i \in[5]$, and $\mathbf{A}$ is generated based on a randomly chosen graph $\mathcal{G}$ from the 
9608 nonisomorphic 5-vertex directed graphs [3] such that $A_{i}=\{j \in[5]:(j, i) \in \mathcal{G}\}$.

- For any $i \in[5], \mathcal{X}_{i}=\{0,1\}$ and $X_{i} \sim \operatorname{Bern}\left(p_{i}\right)$, where $p_{i}$ is uniformly randomly chosen from range $(0,1)$;

- For any $i \in[5], d_{i}=\tilde{d}_{i} \cdot H_{\infty}\left(X_{G_{i}}\right)$, where $\tilde{d}_{i}$ is uniformly randomly chosen from range $(0,1)$;

- $P \subseteq[5]$ is randomly generated assuring that $|P| \leq 2$.

For each system, we compute the lower bounds $\mathcal{L}_{\max }^{\text {Thm. }}$ and $\mathcal{L}_{\max }^{\mathrm{Thm} .2}$ by Theorems 11 and 2 , respectively. An interesting observation is that we have $\mathcal{L}_{\max }^{\mathrm{Thm} .2}>\mathcal{L}_{\max }^{\mathrm{Thm} .1}$ for only 2 among the 500 tested systems, while $\mathcal{L}_{\max }^{\text {Thm. }}{ }^{\text {Th }}>\mathcal{L}_{\max }^{\text {Thm. } 2}$ for all the 498 remaining systems.

We also compute the MaxL according to the privacy mechanism $P_{Y \mid X_{[n]}}$ given by Algorithm 1 , denoted as $\mathcal{L}_{\max }^{\text {Alg.1. Then }}$ we compute the ratio $R=\mathcal{L}_{\max }^{\mathrm{Alg} .1} / \max \left(\mathcal{L}_{\max }^{\mathrm{Thm}} \cdot 1, \mathcal{L}_{\max }^{\mathrm{Thm}} \cdot 2\right)$. A lower ratio $R$ (close to 1 ) means that our converse and achievable results perform well and are quite close to the optimal $\mathcal{L}_{\text {max }}^{*}$, while a higher ratio indicates bad performance. We summarize the values of $R$ from 500 tests in Table \, from which we can see that the proposed techniques achieves a satisfactory level of performance for the majority of tested problems. Notably, we have $\mathcal{L}_{\max }^{\mathrm{Thm} .1}=\mathcal{L}_{\max }^{\mathrm{Alg} .1}$ and thus $R=1$ for 162 among the 500 tests.

Table I

PERFormance of THE LOWER BOUNDS VERSUS ALgORITHM 1

\begin{tabular}{|c|c|c|c|c|c|}
\hline Ratio, $R$ & $=1$ & $<1.05$ & $<1.1$ & $<1.2$ & $\geq 1.2$ \\
\hline $\begin{array}{c}\text { Number of } \\
\text { Systems }\end{array}$ & 162 & 401 & 429 & 460 & 40 \\
\hline
\end{tabular}

Future directions include improving the privacy mechanism and the converse results for the multi-terminal guessing problem (10), as well as studying the multi-terminal privacyutility tradeoff using different privacy and utility measures.

\section{APPENDIX A \\ PROOF OF LEMMA 2}

Note that the right hand side of (5) is equal to the Sibson mutual information of order $\infty$ [21], $I_{\infty}^{\mathrm{S}}$, and hence we have

$$
\begin{aligned}
\mathcal{L}_{\max }\left(X_{Q} \rightarrow Y \mid X_{P}\right) & =I_{\infty}^{\mathrm{S}}\left(X_{Q} ; Y, X_{P}\right) \\
& \geq I\left(X_{Q} ; Y, X_{P}\right)=I\left(X_{Q} ; Y \mid X_{P}\right),
\end{aligned}
$$

where the inequality follows from [22, Theorem 2], and the last equality follows from source independence.

It remains to show $\mathcal{L}_{\max } \geq \Delta_{i}$ for any user $i \in[m]$ with $G_{i} \subseteq Q$. For brevity, we drop the subscript $i$ remembering that $W, A, G$ stand for $W_{i}, A_{i}, G_{i}$, respectively. Set

$$
\begin{aligned}
W_{P} & =P \cap W, & W_{Q} & =Q \cap W, \\
A_{P} & =P \cap A, & A_{Q} & =Q \cap A .
\end{aligned}
$$

Since $G \subseteq Q$, we have $P \cap G=\emptyset, Q \cap G=G$, and thus $P=W_{P} \cup A_{P}$, and $Q=W_{Q} \cup A_{Q} \cup G$. We have

$$
\begin{aligned}
& \mathcal{L}_{\max }\left(X_{Q} \rightarrow Y \mid X_{P}\right) \\
& \geq \sum_{y, x_{P}} P_{Y, X_{P}}\left(y, x_{P}\right) \log \max _{x_{Q}} \frac{P_{Y, X_{P} \mid X_{Q}}\left(y, x_{P} \mid x_{Q}\right)}{P_{Y, X_{P}}\left(y, x_{P}\right)}
\end{aligned}
$$

$$
\begin{aligned}
= & \sum_{y, x_{P}} P_{Y, X_{P}}\left(y, x_{P}\right) \\
& \log \left(\operatorname { m a x } _ { x _ { W _ { Q } } , x _ { A _ { Q } } } \left(\frac{P_{Y, X_{P} \mid X_{W_{Q}}, X_{A_{Q}}}\left(y, x_{P} \mid x_{W_{Q}}, x_{A_{Q}}\right)}{P_{Y, X_{P}}\left(y, x_{P}\right)}\right.\right. \\
& \left.\left.\cdot \max _{x_{G}} \frac{P_{Y, X_{P} \mid X_{Q}}\left(y, x_{P} \mid x_{Q}\right)}{P_{Y, X_{P} \mid X_{W_{Q}}, X_{A_{Q}}}\left(y, x_{P} \mid x_{W_{Q}}, x_{A_{Q}}\right)}\right)\right) \\
= & \sum_{y, x_{P}} P_{Y, X_{P}}\left(y, x_{P}\right) \\
& \log \left(\operatorname { m a x } _ { x _ { W _ { Q } } , x _ { A } } \left(\frac{P_{Y, X_{P} \mid X_{W_{Q}}, X_{A_{Q}}}\left(y, x_{P} \mid x_{W_{Q}}, x_{A_{Q}}\right)}{P_{Y, X_{P}}\left(y, x_{P}\right)}\right.\right. \\
& \left.\cdot \max _{x_{G}} \frac{P_{Y, X_{A} \mid X_{G}}\left(y, x_{A} \mid x_{G}\right)}{P_{Y, X_{A}}\left(y, x_{A}\right)}\right) \\
\geq & \sum_{y, x_{P}, x_{A_{Q}}} P_{Y, X_{P}, X_{A_{Q}}}\left(y, x_{P}, x_{A_{Q}}\right) \\
& \left(\log \max _{x_{W_{Q}}} \frac{P_{Y, X_{P} \mid X_{W_{Q}}, X_{A_{Q}}}\left(y, x_{P} \mid x_{W_{Q}}, x_{A_{Q}}\right)}{P_{Y, X_{P}}\left(y, x_{P}\right)}\right. \\
& \left.+\log _{x_{G}} \frac{P_{Y, X_{A} \mid X_{G}}\left(y, x_{A} \mid x_{G}\right)}{P_{Y, X_{A}}\left(y, x_{A}\right)}\right) \\
= & I\left(Y, X_{P} ; X_{W_{Q}}, X_{A_{Q}}\right) \\
& +\sum_{y, x_{A}} P_{Y, X_{A}}\left(y, x_{A}\right) \log \max _{x_{G}} \frac{P_{Y, X_{A} \mid X_{G}}\left(y, x_{A} \mid x_{G}\right)}{P_{Y, X_{A}}\left(y, x_{A}\right)} \\
\geq & I\left(Y, X_{P} ; X_{W_{Q}}, X_{A_{Q}}\right)+D_{i} \\
= & I\left(Y ; X_{A_{Q}} \mid X_{P}\right)+H\left(X_{W_{Q}}\right)+D_{i}=\Delta_{i},
\end{aligned}
$$

where

- (22) follows from Jensen's inequality;

- (23) follows from that for any $y \in \mathcal{Y}, x_{W \cup A} \in \mathcal{X}_{W \cup A}$,

$$
\begin{aligned}
& \max _{x_{G}} \frac{P_{Y, X_{P} \mid X_{Q}}\left(y, x_{P} \mid x_{Q}\right)}{P_{Y, X_{P} \mid X_{W_{Q}}, X_{A_{Q}}}\left(y, x_{P} \mid x_{W_{Q}}, x_{A_{Q}}\right)} \\
& =\max _{x_{G}} \frac{P_{Y, X_{A \cup G}}\left(y, x_{A \cup G}\right) \cdot P_{X_{W} \mid Y, X_{A \cup G}}\left(x_{W} \mid y, x_{A \cup G}\right)}{P_{X_{G}}\left(x_{G}\right) \cdot P_{Y, X_{A}}\left(y, x_{A}\right) \cdot P_{X_{W} \mid Y, X_{A}}\left(x_{W} \mid y, x_{A}\right)} \\
& \stackrel{(a)}{=} \max _{x_{G}} \frac{P_{Y, X_{A} \mid X_{G}}\left(y, x_{A} \mid x_{G}\right)}{P_{Y, X_{A}}\left(y, x_{A}\right)},
\end{aligned}
$$

where (a) follows from the Markov chain $X_{W}-$ $\left(Y, X_{A}\right)-X_{G}$ as a result of the utility constraint (6);

- (24) follows from replacing maximum over $x_{A_{Q}}$ with expectation over $P_{X_{A_{Q}} \mid Y, X_{P}}$, and Jensen's inequality;

- (25) follows from the fact that $X_{W_{Q}}$ is a deterministic function of $\left(Y, X_{P}, X_{A_{Q}}\right)$ according to (6);

- (26) follows from that

$$
\begin{aligned}
& \sum_{y, x_{A}} P_{Y, X_{A}}\left(y, x_{A}\right) \log \max _{x_{G}} \frac{P_{Y, X_{A} \mid X_{G}}\left(y, x_{A} \mid x_{G}\right)}{P_{Y, X_{A}}\left(y, x_{A}\right)} \\
& \geq \sum_{y, x_{A}} P_{Y, X_{A}}\left(y, x_{A}\right) \log \frac{\max _{x_{G}} P_{Y, X_{A}, X_{G}}\left(y, x_{A}, x_{G}\right)}{\max _{x_{G}} P_{X_{G}}\left(x_{G}\right) \cdot P_{Y, X_{A}}\left(y, x_{A}\right)} \\
& =\mathbb{E}_{P_{Y, X_{A}}}\left[\log r\left(X_{G_{i}} \rightarrow Y \mid X_{A_{i}}\right)\right]=D_{i} ;
\end{aligned}
$$

- 277) follows from source independence, as well as (6). This concludes the proof. 


\section{REFERENCES}

[1] Y. Birk and T. Kol, "Coding on demand by an informed source (ISCOD) for efficient broadcast of different supplemental data to caching clients," IEEE Trans. Inf. Theory, vol. 52, no. 6, pp. 28252830, Jun. 2006.

[2] Z. Bar-Yossef, Y. Birk, T. Jayram, and T. Kol, "Index coding with side information," IEEE Trans. Inf. Theory, vol. 57, pp. 1479-1494, 2011.

[3] F. Arbabjolfaei and Y.-H. Kim, "Fundamentals of index coding," Foun-

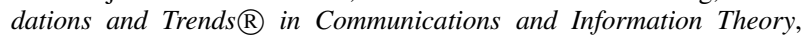
vol. 14, no. 3-4, pp. 163-346, 2018.

[4] S. H. Dau, V. Skachek, and Y. M. Chee, "On the security of index coding with side information," IEEE Trans. Inf. Theory, vol. 58, no. 6 , pp. 3975-3988, 2012.

[5] L. Ong, B. N. Vellambi, P. L. Yeoh, J. Kliewer, and J. Yuan, "Secure index coding: Existence and construction," in Proc. IEEE Int. Symp. on Information Theory (ISIT), Barcelona, Spain, 2016, pp. 2834-2838.

[6] M. M. Mojahedian, M. R. Aref, and A. Gohari, "Perfectly secure index coding," IEEE Trans. Inf. Theory, vol. 63, no. 11, pp. 7382-7395, 2017.

[7] Y. Liu, Y.-H. Kim, B. Vellambi, and P. Sadeghi, "On the capacity region for secure index coding," in Proc. IEEE Information Theory Workshop (ITW), Guanzhou, China, Nov. 2018. [Online]. Available: https://arxiv.org/abs/1809.03615

[8] V. Narayanan, V. M. Prabhakaran, J. Ravi, V. K. Mishra, B. K. Dey, and N. Karamchandani, "Private index coding," in Proc. IEEE Int. Symp. on Information Theory (ISIT), Vail, CO, 2018, pp. 596-600.

[9] Y. Liu, P. Sadeghi, N. Aboutorab, and A. Sharififar, "Secure index coding with security constraints on receivers," arXiv preprint arXiv:2001.07296, 2020.

[10] M. Karmoose, L. Song, M. Cardone, and C. Fragouli, "Privacy in index coding: k-limited-access schemes," IEEE Trans. Inf. Theory, 2019.

[11] I. Issa, A. B. Wagner, and S. Kamath, "An operational approach to information leakage," IEEE Trans. Inf. Theory, 2019.

[12] N. Slonim and N. Tishby, "Agglomerative information bottleneck," in Advances in Neural Information Processing Systems (NIPS), 2000, pp. $617-623$.

[13] A. Makhdoumi, S. Salamatian, N. Fawaz, and M. Médard, "From the information bottleneck to the privacy funnel," in Proc. IEEE Information Theory Workshop (ITW), Hobart, Australia, Nov. 2014, pp. 501-505.

[14] N. Ding and P. Sadeghi, "A submodularity-based clustering algorithm for the information bottleneck and privacy funnel," in Proc. IEEE Information Theory Workshop (ITW), Visby, Sweden, Aug. 2019, pp. $1-5$.

[15] A. Rényi, "On measures of information and entropy," in Proceedings of the 4th Berkeley symposium on mathematics, statistics and probability, vol. 1, no. 547, 1961.

[16] N. Alon, E. Lubetzky, U. Stav, A. Weinstein, and A. Hassidim, "Broadcasting with side information," in 49th Annu. IEEE Symp. on Foundations of Computer Science (FOCS), Oct. 2008, pp. 823-832.

[17] A. Blasiak, R. Kleinberg, and E. Lubetzky, "Lexicographic products and the power of non-linear network coding," in 52nd Annu. IEEE Symp. on Foundations of Computer Science (FOCS), Oct. 2011, pp. 609-618.

[18] F. Arbabjolfaei, B. Bandemer, Y.-H. Kim, E. Sasoglu, and L. Wang, "On the capacity region for index coding," in Proc. IEEE Int. Symp. on Information Theory (ISIT), 2013, pp. 962-966.

[19] R. W. Yeung, Information theory and network coding. Springer Science \& Business Media, 2008.

[20] A. El Gamal and Y.-H. Kim, Network Information Theory. Cambridge: Cambridge University Press, 2011.

[21] R. Sibson, "Information radius," Zeitschrift für Wahrscheinlichkeitstheorie und verwandte Gebiete, vol. 14, no. 2, pp. 149-160, 1969.

[22] S. Verdú, " $\alpha$-mutual information," in Proc. Inf. Theory Appl. Workshop (ITA), Feb. 2015, pp. 1-6. 